\title{
Acute intermittent porphyria
}

\author{
RAYMOND HIERONS \\ M.B., B.Sc., F.R.C.P. \\ Consultant Neurologist, \\ Woolwich, Orpington, Sidcup, Canterbury and East Kent Hospitals
}

PORPHYRINS are an important part of haemoglobin, cytochrome, catalase and, in the plant world, of chlorophyll. They must therefore play a very significant part in a number of vital processes. In health, small amounts can be detected in urine and faeces, while increased amounts are found in a number of pathological conditions such as lead poisoning, liver disease, anaemia, malnutrition and bleeding from the gut. Thus porphyrinuria may be a feature of some acquired disease but porphyria, on the other hand, is the term applied to a constitutional fault or inborn error of metabolism when abnormal quantities and varieties, notably porphobilinogen, coproporphyrin I and II and uroporphyrin are excreted.

There are various types of porphyria and although there is some controversy, it is widely believed that they constitute distinct diseases (Goldberg \& Rimington, 1962).

1. Congenital porphyria is one of the rarest of human diseases. It is characterized by photosensitivity resulting in scarring of the exposed areas and chronic haemolytic anaemia.

2. Porphyria cutanae tarda. Bullae and vesicles develop on the hands and face after exposure to sunlight.

3. Acute intermittent porphyria. This latter variety, although a rare disease, is the commonest type in this country. The incidence has been assessed at about 1 in 80,000 of the population.

A family history is only obtained in a minority of cases because the latent abnormality does not always become manifest during life, but remains symptomless. The mechanism by which the latent condition is converted into disease is often unknown. Sometimes this is precipitated by drugs, particularly barbiturates and sulphonamides, and sometimes by excessive alcohol. Infections may bring on an attack and some have thought an association with pregnancy and even menstruation existed, but this is not certain. Some acute attacks occur for no clear reason.
Probably half the children of an affected individual will have the metabolic defect in a latent form. Transmission is by either sex as a Mendelian dominant character. It is commoner in women than men in the proportion of $3: 2$ although this may only reflect a greater use of barbiturates in women.

Experience suggests that most cases of acute porphyria are not recognized for an excessively long time. Patients, even when very ill, are frequently regarded as neurotic, sometimes even as psychotic. Unnecessary abdominal operations are performed and sedative drugs prescribed which frequently make them worse. Dent (1957) has suggested that as a general rule the diagnosis of inborn errors of metabolism is nearly always a comparatively simple matter because the inborn errors often represent wide deviations from normal'. This is certainly true of porphyria where the clinical features are monotonously stereotyped. The only difficulty is that the diagnosis will not be made unless the possibility comes to mind.

\section{Case report}

In order to illustrate the clinical features I will quote from the notes of a case made before the diagnosis was considered.

Miss W.S., aged 22, was admitted to a teaching hospital on account of abdominal pain of $36 \mathrm{hr}$ duration, associated with vomiting. There was vague tenderness and guarding on abdominal palpation, although a few hours after admission these signs were not confirmed. She had a fever of $99 \cdot 2^{\circ} \mathrm{F}$ and continued to complain of abdominal pain of colicky type and vomited on a number of occasions. The bowel sounds were normal but $\mathrm{X}$-rays of the abdomen showed dilatation of the large bowel. As a result laparotomy was carried out but apart from dilatation of the colon, no abnormality was found.

After the operation the pain continued and she was apathetic and unco-operative. After a few 
days she complained of weakness of the legs, disturbed vision and, a week later, of hallucinations. She was restless at night and required sedation. At this stage a consultant psychiatrist concluded that she was 'an hysteric-emotionally immature and rather dull intellectually-who had been under stress at home and who was now in the ward, beginning to collect additional symptoms from other patients'. She was reassured and it was suggested that when the sutures were removed, her abdominal pain would disappear. This did happen but she then became extremely anxious and tense and had a rapid pulse rate, dry mouth and profuse sweating. She was sent home on the 19th day. After a few days she presented herself at the casualty department complaining of insomnia. Several days later she was brought by ambulance to the psychiatrist, as she was unable to walk or move her arms. She was completely immobile but was again thought to be suffering from conversion hysteria and her own doctor was advised to send her to the local mental hospital.

Another psychiatrist saw her at her home and once more the diagnosis of hysteria was made. On admission to the mental hospital she was described as 'bland and affectless'. She had severe weakness of all four limbs-the arms being more affected than the legs. Apart from the ankle jerks the reflexes were absent. The temperature fluctuated between 99 and $100^{\circ} \mathrm{F}$, and the pulse rate was never less than 110 per minute. She was given sodium amytal each night and a week after admission developed respiratory distress, brought up considerable sputum and was cyanosed. This story, given over the telephone, was enough to suggest the diagnosis and was subsequently confirmed by the presence of large quantities of porphobilinogen in the urine. The only common feature of the disease not mentioned was severe constipation but this was in fact present from the onset.

Over the next 6 months the paresis gradually improved so that she was able to walk when she returned home but it was almost a year before she was back to normal.

About 6 months before her illness, her brother had been operated on for a gastric ulcer and afterwards developed weakness of the legs and a few years later one of the sisters had a similar illness to her own and the same diagnosis was established in these members of the family.

\section{Clinical features}

These can be classified into abdominal, psychological and neurological manifestations. The illness usually develops in this order, with only a severe attack progressing into neurological involvement.

1. Abdominal symptoms are present and prominent in practically every case. Usually these are followed by nervous symptoms but occasionally abdominal symptoms may occur alone and then subside. The onset is usually with intense colicky abdominal pain so that many cases are referred to hospital in the first instance as acute abdominal emergencies. Generally this is associated with severe constipation so that $10-15$ days may pass without a bowel action. Rarely diarrhoea occurs, possibly from impacted faeces. Often there is nausea and vomiting. Abdominal palpation does not reveal tenderness or guarding but abdominal distension may be present. A history of previous attacks of abdominal pain and constipation which subsided after a few days or weeks may be elicited. Many cases are submitted to laparotomy and no abnormality found. Post-operatively the abdominal symptoms are often worse and probably as a result of the thiopentone anaesthetic or barbiturates the condition deteriorates.

2. Psychological manifestations. Insomnia is an almost invariable feature and when sleep comes it is often accompanied by frightening nightmareso $\vec{\varphi}$ During the day the patients are emotional, appres 응 hensive and depressed so that their behavioup is interpreted as hysterical or neurotic. Barbiturates are frequently given, thus producing further aggravation into delirium, hallucinations, either auditory or visual, even delusions and noisy behaviour. It is not surprising that so often these patients are transferred from the surgical wards at this stage to mental hospitals.

Occasionally an attack may terminate at this stage followed by a gradual return to normal. Often if the patient is in hospital and the condition not recognized, with barbiturates continued the third group of symptoms develop.

3. Neurological. These usually start with pain in the back and in the limbs, followed after a few days by the gradual onset of muscular weakness in the arms and legs. This weakness is most marked proximally in the upper limbs but is generalized in the legs and may progress to complete paralysis of all four limbs. The knee jerks are abolished before the ankle jerks but eventually all the tendon reflexes are abolished. Muscle tenderness may be present and wasting may develop at an early stage. In a severe case the patient will not be able to sit up from weakness of the trunk muscles and may be unable to lift the head from the pillow. There may be paralysis of the intercostal and respiratory muscles. On 
rare occasions the plantar responses may be extensor but generally they are flexor.

In the majority of cases the condition is a pure motor polyneuritis without sensory manifestations, but occasionally there is tingling of the hands and feet; rarely sensory loss is detected over the bathing trunk area. Paresis of the palate and vocal cords may cause difficulty with swallowing and inability to speak above a whisper, even hoarseness. Only rarely there is diplopia and weakness of the ocular muscles. During the stage of delirium, tremor may be present and simulate delirium tremens. Occasionally epileptic attacks occur, either generalized or focal.

4. General manifestations. Generally the patient looks ill and loss of weight occurs. A constant feature is tachycardia and this persists for weeks or months. In about half the cases a mild degree of hypertension is observed during the acute attack and this returns to normal with recovery. Only rarely is severe hypertension with papilloedema seen. Oliguria may occur, perhaps in part due to vomiting. Pigmentation of the skin may occur and with the cachexia, Addison's disease may be suggested. Brief episodes of blurred vision, even blindness may occur and Raynaud's phenomenon has been described.

\section{Laboratory investigations}

A trace of protein in the urine may occasionally be present. The serum electrolytes, especially sodium and chloride, are often decreased. This is usually due to vomiting but a lower nephron type of nephrosis may be responsible (Prunty, 1949). Recently an inappropriate secretion of antidiuretic hormone has been found, associated with structural lesions in the hypothalamus (Perlroth et al., 1966). The CSF is usually normal although occasionally the protein and globulin content may be slightly increased.

During an acute attack the urine may be reddish-brown in colour, but in some cases the urine will be normal when first passed, the change in colour occuring only after the urine has been standing for some hours. Similarly coloured urine occurs in other conditions and chemical tests for porphobilinogen are therefore necessary to establish the diagnosis with certainty. This can be estimated on freshly passed urine by the use of Ehrlich's aldehyde reagent or else spectroscopically. Quantitative estimations may be necessary after chromatographic extraction. There is also an increased excretion of $\delta$-aminolaevulinic acid which is a metabolic precursor of porphobilinogen. These abnormalities may or may not persist in the urine after the acute attack has subsided and there is no correlation between the chemical abnormalities and the severity of the symptoms.

\section{Pathology}

There is still much to be worked out about the mechanism of the symptoms and lesions in this disease. Investigations of the intestinal tract during an acute attack suggest that spasm or atony may occur; often a combination of both is present, usually combined with dilatation of the colon. It has been claimed that the application of various porphyrins to the gut or uterus experimentally will produce muscular contraction. Other workers have repeated this and have claimed that neither porphobilinogen nor its precursor $\delta$-aminolaevulic acid are in any way toxic. It is possible, however, that some as yet unidentified vasoconstrictor substance may be produced during the disease. Gajdos \& Gajdos-Török (1961) have suggested that a deficiency of purine synthesis occurs, resulting in a deficiency of other essential metabolities. They have claimed benefit from the administration of adenosine-5-monophosphoric acid in the acute attack.

In a study of the nervous system in fatal cases I have suggested (Hierons, 1957) that the mental disturbances are due to a direct, possibly reversible, metabolic effect of the disease. Vascular lesions can also be found in the brain, probably secondary to severe hyptertension or to respiratory paralysis. The paralysis is explained by demyelination of the peripheral nerves and this is probably due to the metabolic disturbance.

\section{The South African type (Porphyria variegata)}

The disease is extremely common in South Africa. Dean (1963) has suggested an incidence of $1: 300$ of the population. It differs from the European type in that cutaneous manifestations are common. Frequently there is fragility of the skin with sores and scars on the hands and face and often brownish pigmentation of the exposed skin; there may be hirsutism. There are also chemical differences. The European type may have a raised faecal porphyrin level during an acute attack but this becomes normal during a remission. By contrast, in the South African type, the faecal abnormality persists during periods of clinical normality.

\section{Treatment}

Although numerous drugs ranging from steroids to BAL have been advocated, I am doubtful of many of these claims. The complete avoidance of barbiturates, sulphonamides and griseofulvin are the most important measures. For the relief of severe pain, morphia or pethidine may be 
necessary and sedatives such as chlorpromazine or paraldehyde can be used for the nervous symptoms. Adequate fluids and the restoration of electrolyte disturbance will, of course, be necessary. In case of respiratory embarrassment assisted respiration will be life-saving.

Many have noted a relation of acute attacks to ovulation and in this type of case with a relation between menses and symptoms, Perlroth, Marver \& Tschudy (1965) have claimed that the long-term use of oral contraceptive agents can prevent attacks.

The pulse-rate is helpful in assessing recovery and until this is normal remission of the attack has not begun.

However profound the paralysis, full recovery to normality is possible.

\section{References}

DEAN, G. (1963) The Porphyrias. A Story of Inheritance and $\stackrel{\unrhd}{\varrho}$ Environment. Pitman Medical, London.

Dent, C.E. (1957) Foreword. Amer. J. Med. 22, 671.

Gajdos, A. \& Gajdos-ToroK, G. (1961) The therapeutic effect of adenosine-5-monophosphoric acid in porphyria. Lancet, ii, 175.

GoldgerG, A. \& Rimington, C. (1962) Diseases of Porphyrin Metabolism. Thomas, Springfield, Illinois.

Hierons, R. (1957) Changes in the nervous system in acute $\mathbb{D}$ porphyria. Brain, 80, 176.

Perlroth, M.G., Marver, S. \& Tschudy, D.P. (1965) थे Oral contraceptive agents and the management of acute $\overrightarrow{0}$ intermittent porphyria. J. Amer. med. Ass. 194, 1037.

Perlroth, M.G., Tschudy, D.P., Marver, H.S., Berard, C.W., Ziegel, R.F., ReChCIGL, M. \& Collins, A. (1966) Acute intermittent porphyria. New morphologic and biochemical findings. Amer. J. Med. 41, 149.

Prunty, F.T.G. (1949) Sodium and chloride depletion in acute porphyria with reference to the status of adrenal is cortical function. J. clin. Invest. 28, 690. 